Aleksander B. Kharazishvili, Institute of Applied Mathematics, Tbilisi State University, University str. 2, 380043 Tbilisi 43, Republic of Georgia

\title{
ON SELECTORS NONMEASURABLE WITH RESPECT TO QUASIINVARIANT MEASURES
}

\begin{abstract}
We discuss a question on the existence of partial $\mu$-nonmeasurable $H$-selectors, where $\mu$ is a given nonzero $\sigma$-finite measure defined on some $\sigma$-algebra of subsets of a set $E$ and quasiinvariant under an uncountable group $G$ of transformations of $E$, and $H$ is an arbitrary countable subgroup of $G$.
\end{abstract}

Let $E$ be a nonempty set and $G$ be a group of transformations of $E$. Let $S$ be a $\sigma$-algebra of subsets of $E$ and $\mu$ be a measure defined on $S$. We recall that $\mu$ is a $G$-quasiinvariant measure if

a) the $\sigma$-algebra $S$ is a $G$-invariant class of sets;

b) for each $X \in S$ and for each $g \in G$, the equality $\mu(X)=0$ implies the equality $\mu(g(X))=0$.

In particular, every $G$-invariant measure $\mu$ defined on $S$ is simultaneously a $G$-quasiinvariant measure.

Let $H$ be an arbitrary subgroup of $G$. Then, obviously, we have a canonical partition of $E$ consisting of all $H$-orbits.

We say that a subset $Y$ of $E$ is an $H$-selector if $Y$ is a selector of the abovementioned partition. We say that a subset $Y$ of $E$ is a partial $H$-selector if $Y$ is a selector of a subfamily of this partition. Clearly, every partial $H$-selector can be extended to an $H$-selector.

A question on measurability of $H$-selectors, with respect to the given nonzero $\sigma$-finite $G$-quasiinvariant measure $\mu$, arises naturally. We recall that the first result concerning this question was obtained by Vitali [11], who showed that if $E$ is the set of all real numbers, $G$ is the additive group of

Key Words: transformation group, quasiinvariant measure, nonmeasurable selector

Mathematical Reviews subject classification: Primary: 28A05. Secondary: 28A20

Received by the editors June 3, 1996 
reals and $H$ is the additive group of rationals, then each $H$-selector is nonmeasurable with respect to the classical Lebesgue measure on $E$.

This important result of Vitali was generalized in various directions. In particular, some theorems and facts concerning measurability of $H$-selectors, with respect to $\mu$, were obtained in the papers [1], [5], [8] and [9]. Notice that the case where $H$ is an uncountable subgroup of the given group $G$ was discussed in those papers, too.

In the present paper we shall consider only the case when card $(H) \leq$ $\omega$. First of all let us remark that, even in the classical situation, we cannot assert the nonmeasurability of all $H$-selectors. Indeed, in [4] a measure $\nu$ is constructed such that

1) $\nu$ is defined on some $\sigma$-algebra of subsets of the real line;

2) $\nu$ is a nonzero nonatomic $\sigma$-finite measure;

3) $\nu$ is invariant under the group of all isometric transformations of the real line;

4) $\operatorname{dom}(\nu)$ contains the family of all Lebesgue measurable subsets of the real line;

5) there exists a Vitali set belonging to dom $(\nu)$.

We thus conclude that, for the above-mentioned measure $\nu$ and for the countable group $H$ coinciding with the additive group of rationals, there exists a $\nu$-measurable $H$-selector.

In connection with this result it is reasonable to pose the following question.

Let $E$ be a set and $G$ be an uncountable group of transformations of $E$. Let $\mu$ be a nonzero $\sigma$-finite $G$-quasiinvariant measure defined on some $\sigma$-algebra of subsets of $E$ and let $H$ be an arbitrary countable subgroup of the group $G$. Denote by $\{H(x): x \in E\}$ the partition of $E$ into $H$-orbits of points of $E$. Does there exist a subfamily of $\{H(x): x \in E\}$ such that all selectors of this subfamily are nonmeasurable with respect to $\mu$ ?

Our goal is to show that, under some natural assumptions on $G$ and $\mu$, the answer to this question is positive.

We say that the group $G$ acts freely in the space $E$, with respect to the given measure $\mu$, if for any two distinct transformations $g$ and $h$ from $G$, we have the equality

$$
\mu^{*}(\{x \in E: g(x)=h(x)\})=0,
$$

where $\mu^{*}$ denotes, as usual, the outer measure associated with $\mu$.

For example, if $E$ is a finite-dimensional Euclidean space, $G$ is a group of affine transformations of $E$, and $\mu$ is a measure defined on some $\sigma$-algebra of subsets of $E$ and vanishing on all affine hyperplanes of $E$, then $G$ acts freely in $E$ with respect to $\mu$. 
Our further consideration needs the following statement which generalizes a result obtained in [2], [3] and [7].

Theorem 1 Let $E$ be a set, $G$ be a group of transformations of $E$ and let $\mu$ be a nonzero $\sigma$-finite $G$-quasiinvariant measure defined on a $\sigma$-algebra of subsets of $E$. Suppose also that $G$ contains an uncountable subgroup $\Gamma$ acting freely in $E$ with respect to $\mu$. Let $H$ be an arbitrary countable subgroup of $\Gamma$ and let $\{H(x): x \in E\}$ be a partition of $E$ into $H$-orbits. Then there exists a subfamily of $\{H(x): x \in E\}$ such that its union is a $\mu$-nonmeasurable set in $E$.

ProOF. We may assume, without loss of generality, that

a) $\mu$ is a probability measure,

b) the group $\Gamma$ coincides with the original group $G$,

c) $\operatorname{card}(\Gamma)=\operatorname{card}(G)=\omega_{1}$.

Let us denote by $\left\{g_{\xi}: \xi<\omega_{1}\right\}$ a family of elements of $G$ such that

$$
g_{\xi} H \neq g_{\zeta} H \quad\left(\xi<\omega_{1}, \zeta<\omega_{1}, \xi \neq \zeta\right) .
$$

The existence of such a family is obvious since card $(G)=\omega_{1}$ and card $(H) \leq \omega$. Next, since $H$ is a countable group, we can write $H=\left\{h_{n}: n \in \omega\right\}$. Let $F$ be a subset of $E$ for which the family $\{G(y): y \in F\}$ is injective and consists of all $G$-orbits in $E$ (in other words, $F$ is a $G$-selector). Consider a family $\{H(y): y \in F\}$ and put $Y=\cup\{H(y): y \in F\}$. If the set $Y$ is nonmeasurable with respect to $\mu$, then there is nothing to prove. Suppose now that $Y \in \operatorname{dom}(\mu)$. Then it is not hard to check that, for any two distinct ordinals $\xi<\omega_{1}$ and $\zeta<\omega_{1}$, we have the inclusion

$$
g_{\xi}(Y) \cap g_{\zeta}(Y) \subseteq g_{\xi}\left(\cup\left\{h_{n}\left(X_{n m}\right): n \in \omega, m \in \omega\right\}\right),
$$

where a set $X_{n m}$ is defined by the formula

$$
X_{n m}=\left\{x \in E: g_{\xi} h_{n}(x)=g_{\zeta} h_{m}(x)\right\} .
$$

But $\mu^{*}\left(X_{n m}\right)=0$, since $G$ acts freely in $E$ with respect to $\mu$. Taking into account the fact that $\mu$ is a $G$-quasiinvariant measure, we obtain

$$
\mu\left(g_{\xi}(Y) \cap g_{\zeta}(Y)\right)=0,
$$

for all $\xi<\omega_{1}, \zeta<\omega_{1}, \xi \neq \zeta$. The latter relation implies the equality $\mu(Y)=0$, since $\mu$ (being a $\sigma$-finite measure) satisfies the countable chain condition.

Now, it is easy to see that we can represent the set $E$ in the form

$$
E=\cup\left\{Y_{\alpha}: \alpha<\omega_{1}\right\}
$$


where

1) the sets $Y_{\alpha}\left(\alpha<\omega_{1}\right)$ are pairwise disjoint,

2) for each $\alpha<\omega_{1}$, the set $Y_{\alpha}$ is the union of a family of $H$-orbits in $E$,

3) for each $\alpha<\omega_{1}$, we have $\mu\left(Y_{\alpha}\right)=0$.

According to the classical theorem of Ulam [10], there exists a subset $A$ of $\omega_{1}$ such that the set $\cup\left\{Y_{\alpha}: \alpha \in A\right\}$ does not belong to $\operatorname{dom}(\mu)$. But it is clear that $\cup\left\{Y_{\alpha}: \alpha \in A\right\}$ can be represented as the union of a family of $H$-orbits in $E$. Thus, the theorem is proved.

Now, we can easily deduce from Theorem 1 the following statement.

Theorem 2 Let $E$ be a set and $G$ be an uncountable group of transformations of $E$. Let $\mu$ be a nonzero $\sigma$-finite $G$-quasiinvariant measure defined on some $\sigma$-algebra of subsets of $E$. Suppose that $G$ acts freely in $E$ with respect to $\mu$. Fix a countable subgroup $H$ of $G$ and denote by $\{H(x): x \in E\}$ the partition of $E$ consisting of all $H$-orbits. Then there exists a subfamily of $\{H(x): x \in E\}$ such that all its selectors are nonmeasurable with respect to $\mu$.

Proof. According to Theorem 1, there exists a subset $D$ of $E$ such that the family $\{H(x): x \in D\}$ is injective and the set $\cup\{H(x): x \in D\}$ is nonmeasurable with respect to $\mu$. Let us show that all selectors of $\{H(x)$ : $x \in D\}$ are $\mu$-nonmeasurable, too. Denote by $Z$ an arbitrary selector of $\{H(x): x \in D\}$. Obviously, we have the equality

$$
\cup\{H(x): x \in D\}=\cup\{h(Z): h \in H\} .
$$

Suppose that $Z \in \operatorname{dom}(\mu)$. Then, taking into account the fact that $H$ is a countable group and $\mu$ is a $G$-quasiinvariant measure, we obtain

$$
\cup\{h(Z): h \in H\} \in \operatorname{dom}(\mu)
$$

and, consequently,

$$
\cup\{H(x): x \in D\} \in \operatorname{dom}(\mu),
$$

which contradicts the definition of the family $\{H(x): x \in D\}$. This contradiction finishes the proof.

Remark 1 It is essential for validity of Theorem 2 that the partition $\{H(x)$ : $x \in E\}$ of the set $E$ consists of all $H$-orbits, where $H$ is a countable subgroup of the original transformation group $G$. In order to show this, let us take an arbitrary group $G$ with $\operatorname{card}(G)=\omega_{1}$ and let us put $E=G$. Clearly, we can 
identify $G$ with the group of all left translations of $E$, which acts freely in $E$. Further, we can represent $G$ in the form

$$
G=\cup\left\{G_{\xi}: \xi<\omega_{1}\right\}
$$

where a family $\left\{G_{\xi}: \xi<\omega_{1}\right\}$ satisfies the following conditions:

1. for each $\xi<\omega_{1}$, we have $\operatorname{card}\left(G_{\xi}\right)=\omega$,

2. for each $\xi<\omega_{1}$, the set $G_{\xi}$ is a subgroup of the group $G$,

3. for each $\xi<\omega_{1}$, the set $\cup\left\{G_{\zeta}: \zeta<\xi\right\}$ is a proper subset of $G_{\xi}$.

Let us fix a point $e \in E$ and let us put

$$
E_{\xi}=G_{\xi}(e) \backslash \cup\left\{G_{\zeta}(e): \zeta<\xi\right\},
$$

for all ordinals $\xi<\omega_{1}$. Then we obtain a partition $\left\{E_{\xi}: \xi<\omega_{1}\right\}$ of the set $E$ such that card $\left(E_{\xi}\right)=\omega$, for any $\xi<\omega_{1}$. We assert now that an analogue of Theorem 2 is not true for the above-mentioned partition. Indeed, let $\lambda$ be a probability diffused measure defined on the $\sigma$-algebra of subsets of the set $E$, generated by the family of all countable subsets of $E$, and let $Z$ be a fixed selector of $\left\{E_{\xi}: \xi<\omega_{1}\right\}$. Denote by $J$ the $G$-invariant $\sigma$-ideal of subsets of $E$, generated by the one-element family $\{Z\}$. It is easy to check that, for any set $X \in J$, we have the equality $\lambda_{*}(X)=0$, where $\lambda_{*}$ denotes, as usual, the inner measure associated with $\lambda$. Starting with this property of $J$ we can easily extend the measure $\lambda$ to a measure $\mu$ such that

a) $\operatorname{dom}(\mu)$ coincides with the $\sigma$-algebra of subsets of $E$, generated by $\operatorname{dom}(\lambda) \cup J$

b) $\mu(X)=0$ for all sets $X \in J$,

c) $\mu$ is a $G$-invariant measure.

Now, it is clear that, for any subset $\Xi$ of $\omega_{1}$, there exists a $\mu$-measurable selector of the family $\left\{E_{\xi}: \xi \in \Xi\right\}$.

A similar argument shows us that if $H$ is an arbitrary uncountable subgroup of our group $G$ and $\{H(x): x \in E\}$ is a partition of $E$ into $H$-orbits, then for every selector $Z$ of $\{H(x): x \in E\}$ there exists a measure $\nu$ satisfying the following conditions:

(1) $\nu$ is a complete probability diffused $G$-invariant measure defined on some $\sigma$-algebra of subsets of $E$,

(2) $Z$ belongs to $\operatorname{dom}(\nu)$ and $\nu(Z)=0$.

In particular, we obtain immediately from (2) that, for each subset $F$ of $E$, there is a selector of $\{H(x): x \in F\}$ belonging to $\operatorname{dom}(\nu)$.

The next result is an easy consequence of Theorem 2 . 
Proposition 1 Let the assumptions of Theorem 2 be satisfied and let, in addition, a countable subgroup $H$ of $G$ be such that card $(H(x)) \geq 2$, for all points $x \in E$. Then there exists an $H$-selector nonmeasurable with respect to $\mu$.

Proof. Indeed, it immediately follows from Theorem 2 that there exists a partial $H$-selector $Z$ nonmeasurable with respect to $\mu$. Evidently, we can find two $H$-selectors $Z_{1}$ and $Z_{2}$ which extend $Z$ and satisfy the equality $Z=Z_{1} \cap Z_{2}$. Now, since the set $Z$ is $\mu$-nonmeasurable, at least one of the sets $Z_{1}$ and $Z_{2}$ is $\mu$-nonmeasurable. Thus, we see that there exists a $\mu$-nonmeasurable $H$ selector.

In fact, the preceding argument shows that, for a measure $\mu$ defined on some $\sigma$-algebra of subsets of a set $E$, the following two assertions are equivalent:

a) there exists a subset of $E$ nonmeasurable with respect to $\mu$,

b) if $\left\{E_{i}: i \in I\right\}$ is a partition of $E$ such that $2 \leq \operatorname{card}\left(E_{i}\right) \leq \omega$, for all $i \in I$, then there exists a selector of $\left\{E_{i}: i \in I\right\}$ nonmeasurable with respect to $\mu$.

Remark 2 Let $E$ be a set and let $G$ be an uncountable group of transformations of $E$, acting freely in $E$ with respect to a nonzero $\sigma$-finite $G$-invariant measure $\mu$ defined on some $\sigma$-algebra of subsets of $E$. It was proved in [9] that there always exists a countable subgroup $H$ of $G$ such that all $H$-selectors are nonmeasurable with respect to every $G$-invariant measure extending $\mu$. In other words, the group $H$ plays a role similar to the role played by the additive group of rationals in the classical Vitali construction [11]. In connection with this result, we wish to notice that the method of [9] is essentially based on the assumption of $G$-invariance of the measure $\mu$ and, therefore, it does not work for nonzero $\sigma$-finite $G$-quasiinvariant measures.

Remark 3 Let $(G,+)$ be an uncountable commutative group equipped with a nonzero $\sigma$-finite $G$-quasiinvariant measure $\mu$. It was shown in [6] that there always exists a $\mu$-nonmeasurable subgroup of $G$. Starting with this result it is not difficult to prove that, if $H$ is an arbitrary countable subgroup of $G$, then there exists a family of $H$-orbits whose union is a $\mu$-nonmeasurable subgroup of $G$. Notice also that an analogous assertion is not true, in general, for uncountable noncommutative groups (see [6]).

Remark 4 It is easy to see that the results presented above can be formulated and proved in a more general form, namely, in terms of the pair $(S, J)$, where

1) $S$ is a $G$-invariant $\sigma$-algebra of subsets of $E$,

2) $J$ is a $G$-invariant $\sigma$-ideal of subsets of $E$, 
3) $J$ is contained in $S$,

4) $(S, J)$ satisfies the countable chain condition.

In particular, we have the respective analogues of Theorems 1, 2 and Proposition for the Baire property.

\section{References}

[1] J. Cichoń, A. B. Kharazishvili and B. Weglorz, On sets of Vitali's type, Proc. Amer. Math. Soc., 118 (1993), 1221-1228.

[2] P. Erdös and R. D. Mauldin, The nonexistence of certain invariant measures, Proc. Amer. Math. Soc., 59 (1976), 321-322.

[3] A. B. Kharazishvili, Certain types of invariant measures, Dokl. Akad. Nauk SSSR, 222(3) (1975), 538-540, (in Russian).

[4] A. B. Kharazishvili, Some applications of Hamel bases, Bull. Acad. Sci. Georgian SSR, 85(1) (1977), 17-20 (in Russian).

[5] A. B. Kharazishvili, Martin's axiom and $\Gamma$-selectors, Bull. Acad. Sci. Georgian SSR, 137(2) (1990),(in Russian).

[6] A. B. Kharazishvili, Selected Topics of Point Set Theory, Łódź University Press, Łódź, 1996.

[7] C. Ryll-Nardzewski and R. Telgarsky, The nonexistence of universal invariant measures, Proc. Amer. Math. Soc., 69 (1978), 240-242.

[8] S. Solecki, On sets nonmeasurable with respect to invariant measures, Proc. Amer. Math. Soc., 119(1) (1993), 115-124.

[9] S. Solecki, Measurability properties of sets of Vitali's type, Proc. Amer. Math. Soc., 119(3) (1993), 897-902.

[10] S. Ulam, Zur Masstheorie in der allgemeinen Mengenlehre, Fund. Math., 16 (1930), 140-150.

[11] G. Vitali, Sul Problema della Misura dei Gruppi di Punti di una Retta, Bologna, Italy, 1905. 